\title{
The Effect of Behavioral Strategies on Nutritional Health
}

\author{
Ruqia Ahmed Sheikh Nur and Ayesha Mushtaq* \\ Dietitian, Pakistan \\ *Corresponding Author: Ayesha Mushtaq, Dietitian, Pakistan.
}

Received: October 28, 2019; Published: November 05, 2019

DOI: $10.31080 /$ ASNH.2019.03.0527

\begin{abstract}
A behaviour strategy has direct effect on nutritional health. Many aspects of eating behaviour help to recognize effective measure of the treatment and the prevention of diseases like obesity, diabetes, or the metabolic syndrome. Whose natural history is strongly influenced by nutritional factors. Factors such as education level and income determine food choices and behaviour in a way that does affect the risk of obesity. Behaviour treatment has been observed to be very useful in weight loss. Attention is very important to expand effective maintenance intervention and searching ways to development of obesity. Behavioral strategies that is useful in the treatment and prevention of hypertension, dyslipidemia and some forms of cancer.
\end{abstract}

Keywords: Nutritional Health; Nutrition

\section{Introduction}

Overweight and obesity are the most important public health concern globally. While non-modified mechanism e.g genetic are to be partially held accountable as obesity runs in the family. Food plays a vital role in a person's age between 10 and 18 years. Nutrition advisory approaches are based on action plans or strategies outlined to achieve the role of behavioral diversity toward a specific client's target. Each concept of change behavior provides the framework or concepts that tried to clarify behavioral variations and integrate information about progressions of behavioral variety (e.g., self-efficiency or ability, change steps) which may be relevant modificational behavior or attitude change. Concepts and methods consistently suggest strategies that emphasize aspects of the change process to endorse variation of desired behave. There are certain strategies used in many concepts and methods. Goal setting is a approach supported or promoted by both cognitive behavioral therapy (CBT) and theory of social cognitive (SCT). Food professionals use a variety of policies based on the client's aims and dietary consulting style and personal accomplishment or talent. Moreover, Nutrition Care Scheme, professionals document the strategies used in nutritional advising along with the auditor of successfully for the policies of nutritional advisory (eg, self-regulating and reduction weight).

The Nutrition Advisory Working by cluster reviewed the evidence nutritional problems and they performed flowing procedures to improve quality life and their diet habits. In addition, nutritional advisory policies are: motivational or inspiring interviewing, self-regulating, uses dietary alternatives, using dietary alternatives and/or structured dietary action, solution of the problem, community support, setting target or objectives, retraining. Cognitive adjustments, control of the stress and stimulus regulator [1].

\section{Motivational interviewing}

Incentive interviewing is a patient-centric strategy designed to bring about change behavior through helping clients to explore or examine and solve changing environments Nutrition professionals regularly use motivational interviewing when using trans-thinking method with client pattern that are pre-contemplative, meditative and planning phases or levels that necessity attention- focused 
interventions to decide the agenda using compassion, discrimination, support, inspiration, and active listening behaviors. The complete questionnaire, reflective listening, clarification, and summary were used to aid the client explore and resolution ambivalence and challenges in behavior change. Practice in motivational interviewing is extremely encouraged by working with clients who are not in the action step of variation [2].

\section{Self-monitoring}

Self-monitoring is used in cognitive behavioral therapy and social cognitive theory or concept and influenced the client having a record of opinions, feelings, eating habits, physical activity, and health measures (e.g., blood sugar, hypertension). The database was reviewed by the client to generate a sand form and was used to help solve the problems and set the goals. Three RCTs have been reviewed and given strong evidence that dietary consumption of selfmonitoring enhance nutritional results that associated reduction of weight and obedience to kidney diets. Three observational studies have shown that clients registered in appropriate weight loss programs for self-monitoring have significantly succeeded in weight loss $[3,4]$.

\section{Reward strategies}

Reward policies influence a systematic procedure in which a professional or client uses rewards to promote particular behavioral variety. In nutritional advisory, rewards can be used for presence, completion of diet records, reduction of weight, or can be pre-defined. Financial monitoring provide for aptitude acquisition or losing weight has not treatment effect in a two RCTs and one of methodological review (including seven RCTs) [5,6].

\section{Problem solving}

Problem solving techniques are often used in cooperation with clients and include recognizing fences to reaching objectives, brainstorming solutions, weighing the pros and cons of possible clarifications or solution, assessing effective solutions and improving strategies.

According solving there's two high-quality RCTs, one overweight and obese female and the other women in postmenopausal along with diabetes females, intervention services combined with problem-solving strategies was used. Furthermore, in both studies, the use of problem solving policies resulted in sustained weight. In subjects with diabetes, the use of problem solving policies has been related with fat intake, self-usefulness, and physical activity $[7,8]$.

\section{Social support}

Social support is capability to construct and use a network or system of household, friends, coworkers, and health practitioners for information or data, inspiration, emotional assist, and promoting an environment that assist cultural diversity. Dietary professionals can be help customers using this approach through building a partnership with the client, assist a client to demonstrate importance of the family and motivation of the public or social support and make client training a on how to successfully elicit this helping or support. Evaluating of the impact of community support on diet associated with behavioral change and it contains different measures of community support assessed by variety of equipment's and their principles. Some researchers conducted their studies 1990s to evaluated the impact of partner and inclusion of the friend on a behavioral modification therapy and found no therapeutic impact or effect. In addition, extreme variation of lifestyle found community support was beneficial to arbitrate the intervention of the impact on physical activity, fat intake, and alteration of HbA1C. Further research is needed to evaluate the effects of different types of community or public support (e.g., perceived public support, value and size of public network, or emotional help) on the particular kinds of objective behaviors variety with different populations [9].

\section{Cognitive restructuring}

Cognitive rehabilitation is a approach often used by nutritional advisors to enhance the awareness of client about their own thoughts and beliefs associated diet, overweight and underweight. Because the use of cognitive rehabilitation for patients with eating disorders was omitted from the review, cognitive rehabilitation as an independent variable was initiated in only one study. In this RCT, the effect of part of the restructuring of a 10-week substanceweight loss program was tested in 63-year-old, high-fat subjects. There was no significant variance between the treatment and control group for physical, behavioral, and/or cognitive events at baseline, after treatment, and 3 month of check-out. Further research is needed to eliminate the impacts of re-organizing knowledge or cognitive as part of a behavioral interference on behaviors related to nutrition [10].

\section{Stress management}

Anxiety management guidelines that focus on environmental stress (e.g., guiding planning or applying time organizing experience) and stressful stress (e.g., using good communication or relaxation exercises) are certain time used in nutritional advisory condi- 
tion There was no literature published in the period 1986-2007 on the time to evaluate the impact of stress management policies on nutrition linked results or outcomes [1].

Stimulus control

Movement control is a fundamental strategy used in behavioral therapy that encompasses altering social or environmental events or stimulating negative behaviors related to diet and exercise. In accordance with the theory of complex behavior, special attention is paid to the strengthening and rewarding effects of environmental change. No new literature has given evidence on the use of nutritional suppression controls associated the outcomes or results has been published in the previous 20years [1].

\section{Conclusion}

A behaviour strategy has direct effect on nutritional health. Many aspects of eating behaviour help to recognize effective measure of the treatment and the prevention of diseases like obesity, diabetes, or the metabolic syndrome. Overweight and obesity are the most important public health concern globally. While nonmodified mechanism e.g. genetic are to be partially held accountable as obesity runs in the family. Food plays a vital role in a person's age between 10 and 18 years. Reward policies influence a systematic procedure in which a professional or client uses rewards to promote particular behavioral variety. In nutritional advisory, rewards can be used for presence, completion of diet records, reduction of weight, or can be pre-defined. Dietary professionals can be help customers using this approach through building a partnership with the client, assist a client to demonstrate importance of the family and motivation of the public or social support and make client training a on how to successfully elicit this helping or support. No new literature has given evidence on the use of nutritional suppression controls associated the outcomes or results has been published in the previous 20years. The whole study shows that diet have great impact on behavior strategy and also behavior strategy have more impact on nutritional health because habits shows your actions towards everything.

\section{Bibliography}

1. International Dietetics and Nutrition Terminology (IDNT) Reference Manual: Standardized Language for the Nutrition Care Process. $2^{\text {nd }}$ ed. Chicago, IL: American Dietetic Association (2009).

2. Miller WR and Rollnick S. "Motivational Interviewing: Preparing People for Change". 2nd ed. New York, NY: Guilford Press (2002).
3. Mattfeldt-Beman MK., et al. "Participants' evaluation of a weight-loss program". Journal of the American Dietetic Association 99 (1999): 66-71.

4. Streit KJ., et al. "Food records: A predictor and modifier of weight change in a long-term weight loss program". Journal of the American Dietetic Association 91 (1991): 213-216.

5. Ditschuneit $\mathrm{HH}$ and Flechter-Mors M. "Value of structured meals for weight management: Risk factors and long-term weight maintenance". Obesity Research 9 (2001): 284S-289S.

6. Paul-Ebhohimhen V and Avenell A. "Systematic review of the use of financial incentives in treatments for obesity and overweight". Obesity Review 23 (2007): 1-13.

7. Glasgow RE., et al. "Assessment of problem-solving: A key to successful diabetes self-management". Journal of Behavior Medicine 27 (2004): 477-490.

8. Perri MG., et al. "Relapse prevention training and problemsolving therapy in the long-term management of obesity". Journal of Consulting and Clinical Psychology 69 (2001): 722-726.

9. Barrera M., et al. "Social support and social-ecological resources as mediators of lifestyle intervention effects for type 2 diabetes". Journal of Health Psychology 11 (2006): 483-495.

10. DeLucia JL and Kalodner CR. "An individualized cognitive intervention: Does it increase the efficacy of behavioral interventions for obesity?" Addictive Behavior 15 (1990): 473-479.

\section{Volume 3 Issue 12 December 2019 (C) All rights are reserved by Ruqia Ahmed Sheikh Nur and Ayesha Mushtaq.}

